\title{
Inductive Substituent Effects: Metal Surfaces versus the Gas Phase ${ }^{\dagger}$
}

\author{
Mark T. Buelow and Andrew J. Gellman* \\ Department of Chemical Engineering, Carnegie Mellon University, Pittsburgh, Pennsylvania 15213
}

Received: July 29, 1999; In Final Form: January 19, 2000

\begin{abstract}
The influence of metal surfaces on inductive (or electrostatic field) substituent effect measurements for surface reactions has been evaluated using image charge theory. Substituent effects have been a valuable tool for determining the electronic properties of transition states for reactions in many environments but have not received much use or consideration on metal surfaces. An important mechanism by which substituents can alter the activation barrier of a reaction is through local dipole field effects on a charged reaction center. To evaluate the influence of a nearby metal surface, substituent field effects have been modeled by considering a point charge and a dipole, both positioned above a conductive surface, that interact electrostatically with their corresponding images at equal distances below the surface. It has been found that the magnitude of substituent effects for reactions on metal surfaces will be approximately equal to the gas-phase value for geometries in which the substituent is farther from the surface than the reaction center and both are above the image plane. This model was used to describe $\beta$-hydride elimination from ethoxides on $\mathrm{Cu}(111)$. This reaction has been found to have a substituent effect that correlates with the reaction energetics of gas-phase alcohol dehydrogenation, a result that is in agreement with the image charge model of the electrostatic influence of the metal.
\end{abstract}

\section{Introduction}

For a given reaction that is studied using a set of reactants having different substituents, the reaction energetics will depend on the nature of the substituent. For example, in a reaction such as the dehydrogenation of alcohols $\left(\mathrm{RCH}_{2} \mathrm{OH} \rightarrow \mathrm{RCH}=\mathrm{O}+\right.$ $\mathrm{H}_{2}$ ), the substituent " $\mathrm{R}$ " can be one of many different functional groups. Measurement of substituent effects on the energetics of a given reaction is used in physical organic chemistry to gain information about that chemical process. ${ }^{1-3}$ The substituent is simply a part of the molecule that does not undergo change during the reaction but does influence the overall reaction energetics. The effect of the substituent can be electronic (electrostatic field effects, polarizability effects, or resonance effects) or physical in nature (steric effects). Correlation of the changes in reaction energetics with substituent properties provides valuable information about the process of interest. For example, correlations between the activation energy for a reaction and field properties of the substituents can be used to probe the change in charge distribution between the reactant and the transition state. As such, substituent effects have been used to determine the nature of the transition states for several surface reactions. ${ }^{4-8}$ For example, for $\beta$-hydride elimination in ethoxides on $\mathrm{Cu}(111)\left(\mathrm{RCH}_{2} \mathrm{O}_{\mathrm{ad}} \rightarrow \mathrm{RCH}=\mathrm{O}+\mathrm{H}_{\mathrm{ad}}\right.$, where $\mathrm{R}$ $=\mathrm{CH}_{3}, \mathrm{CFH}_{2}, \mathrm{CF}_{2} \mathrm{H}$, or $\mathrm{CF}_{3}$ ), it was found that increasing the amount of fluorine at the methyl group led to an increase in the activation barrier. Because the electronegativity of fluorine systematically increases the inductive character of the substituted methyl group, this result indicates that the transition state to breaking the $\beta$ - $\mathrm{CH}$ bond is positively charged (or electron deficient) with respect to the reactant ethoxide $(\mathrm{RC}-\mathrm{H} \rightarrow$ $\left.\left[\mathrm{RC}^{\delta+}+\cdot \mathrm{H}\right]^{\ddagger}\right){ }^{4}$

The nature of substituent interactions with the reaction center

\footnotetext{
† Part of the special issue "Gabor Somorjai Festschrift".

* To whom correspondence should be addressed.
}

has been described for gas-phase processes ${ }^{1}$ and, in lesser detail, for solution-phase reactions; ${ }^{2,3}$ however, no analysis of the mechanism of substituent effects on metal surfaces has been described. We have used a simple image charge model of the metal surface to analyze the magnitude of substituent effects on metals. Specifically, we have considered field effects (also known as inductive effects), because such effects are an important substituent interaction mechanism that has been used experimentally to probe the transition states of several surface reactions. The principle behind this substituent interaction mechanism is that a local dipole moment that is associated with a substituent interacts electrostatically with the changing charge distribution at the reaction center. This electrostatic interaction will raise or lower the activation barrier, depending on whether it is repulsive or attractive. If one measures the activation energies for a reaction in a set of molecules having substituents with differing local dipole moments, one can determine whether the reaction center in the transition state is positive, negative, or uncharged with respect to the initial state. The nearby presence of an infinitely polarizable metal surface must, in principle, affect the magnitude of these electrostatic interactions. This article describes the influence of a metal surface on the magnitude of substituent effects for surface reactions by using a very simple image charge theory to describe the influence of the metal.

The results obtained from our electrostatic model of substituent effects will be compared to an important surface reaction that exhibits a significant substituent effect. $\beta$-hydride elimination in ethoxides on the $\mathrm{Cu}(111)$ surface has been found to have a very large substituent effect, $\Delta \Delta \mathrm{E}_{\text {act }}=55 \mathrm{~kJ} / \mathrm{mol}$, on going from ethoxide $\left(\mathrm{CH}_{3} \mathrm{CH}_{2} \mathrm{O}_{\mathrm{ad}}\right)$ to trifluoroethoxide $\left(\mathrm{CF}_{3} \mathrm{CH}_{2} \mathrm{O}_{\mathrm{ad}}\right){ }^{4}$ The increase in activation energy as a result of fluorination indicates that, with respect to the initial state, the reaction center is electron deficient in the transition state. One important characteristic of $\beta$-hydride elimination in the adsorbed ethoxides 


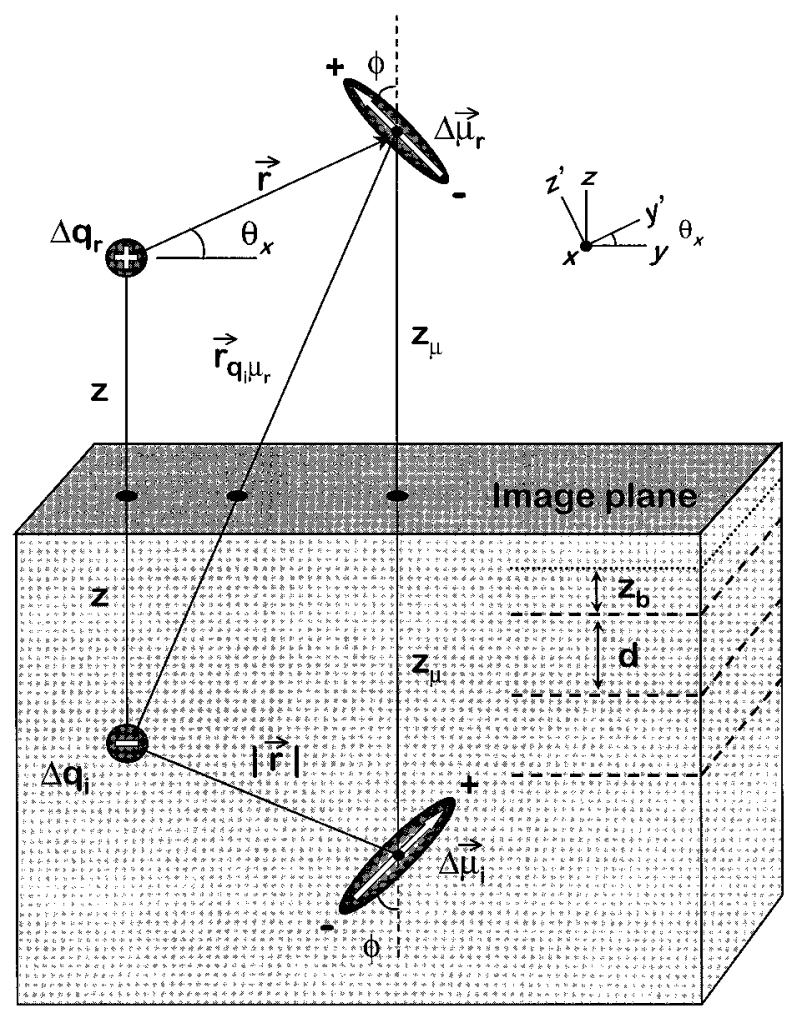

Figure 1. Schematic of the model for the electrostatic interaction of a point charge (reaction center), dipole (substituent), and their images below a metal surface defined by the image plane. All objects lie in the same plane $(y z)$. The independent parameters chosen to describe the geometry are $z, r, \theta_{x}, \phi$, and $\theta_{z}$. The other dependent parameters, defined for convenience, are $r_{q_{\mathrm{i}} \mu_{\mathrm{r}}}, r_{q_{\mathrm{r}}} \mu_{\mathrm{i}}$, and $z_{\mu}$. The dashed lines represent the spacing of the nuclear planes, and the dotted line is the edge of the uniform positive background at a position $d / 2$ above the plane of the surface nuclei. Real objects have the subscript $r$, and images have the subscript $\mathrm{i}$.

is that the geometry of the reactants is well-characterized. ${ }^{9,10}$ Furthermore, the energetics of this reaction have been compared to those for gas-phase dehydrogenation of the corresponding alcohols, and the substituent effects have been shown to be comparable in magnitude. ${ }^{11}$ Given the potential for charge screening by the metal surface, this finding might seem surprising, and it has motivated our comparison of field substituent effects on metal surfaces with those observed in the gas phase.

\section{Model}

The electrostatic model of substituent effects on metal surfaces is described below and illustrated schematically in Figure 1. For the surface reactions and substituents studied in our previous work, the primary mechanism by which substituents interact with the reaction center is through field effects. ${ }^{4-8}$ A point charge, $\Delta q$, represents the change in charge density at the reaction center. As an example, in the case of $\beta$-hydride elimination in ethoxides, the carbon atom in the $\beta$-position with respect to the surface becomes electron deficient $(\Delta q>0)$ on going from reactant ethoxide to the transition state $(\mathrm{RC}-\mathrm{H} \rightarrow$ $\left.\left[\mathrm{RC}^{\delta+} \cdot . \mathrm{H}\right]^{\ddagger}\right) .{ }^{4}$ The substituents, $\mathrm{R}$, have local dipole vectors of magnitude, $\mu$. In the case of $\beta$-hydride elimination in ethoxides, the substituents are fluorinated methyl groups adjacent to the reaction center, and they influence the activation energy $\left(\Delta E_{\mathrm{a}}\right)$. It is the differences in the dipole moments, $\Delta \vec{\mu}$, among the substituents that influences the activation energy. In a set of molecules, all experiencing the same reaction, substituent effects are the changes in activation energy $\left(\Delta \Delta E_{\mathrm{a}}\right)$ that result from the electrostatic interaction between a change in charge $(\Delta q)$ at a reaction center and differences in dipole moments $(\Delta \vec{\mu})$ among the substituents. Thus, in Figure 1, we have depicted the substituent as a dipole of magnitude $\Delta \mu$ at a fixed distance, $r$, from the point charge, $\Delta q$, representing the reaction center.

Image charge theory has been used to describe the electrostatic interactions of the reactant and the transition state with the metal surface. The basis for the method of images is that the electric field at a metal surface can only be perpendicular to the surface and that no electric field exists below the surface. ${ }^{12}$ For a point charge above a surface, this condition gives rise to an electric field above the surface that is the same as the field that would exist if there were an image charge of opposite sign at an equal distance below the surface. When a charge is placed very near the metal surface, the appropriate location of the image plane is not clear and must be considered. Compelling SCFbased calculations of $\mathrm{Pt}_{25}$ clusters indicate that, at the atomic scale, the best choice of image plane is one that varies with the position of the charge over the surface. ${ }^{13}$ Unfortunately, the exact position depends on the response of the metal to the charge, which is a function of the properties of the metal, the magnitude of the charge, and whether the charge is positive or negative. Because of this complexity and in order to gain a semiquantitative view of the effect of the metal on substituent effects, we shall choose a simpler model that is based on an image plane at a constant height from the surface, with a location that is determined on the basis of density-functional calculations of the uniform background model. ${ }^{14-16}$ The edge of the uniform positive background, $z_{\mathrm{b}}$, which represents the potential of the atomic nuclei, is positioned at a height of $d / 2$ above the plane defined by the surface nuclei, where $d$ is the interatomic spacing. For the (111) plane of an fcc metal $d=a / \sqrt{3}$, where $a$ is the lattice constant. The lattice constant for copper is $3.651 \AA$, and thus, $z_{\mathrm{b}}=1.0 \AA$ beyond the atomic nuclei plane. ${ }^{17}$ The electron density extends past this uniform background edge, and the mean of the electron density is located at approximately $\left(z_{0}-\right.$ $\left.z_{\mathrm{b}}\right)=0.8 \AA$ for a transition metal such as $\mathrm{Cu} .{ }^{15,16}$ Thus, the image plane is approximately $1.8 \AA$ from the surface plane defined by the atomic nuclei.

The configuration of the point charge (reaction center), dipole (substituent), and the image plane is best visualized by examining Figure 1. It is important to remember that the surface being depicted is the image plane and not the plane defined by the atomic nuclei. The positions of the nuclear planes are indicated with the dashed lines, and the position of the uniform background edge is indicated with the dotted line. Two coordinate systems will be used to describe the geometry: a surface coordinate system $(x, y, z)$ and a molecular coordinate system $\left(x, y^{\prime}, z^{\prime}\right)$. The $x$ axis is the same in both coordinate systems and projects out of the plane of Figure 1. For the surface coordinates, the $z$ axis is the surface normal, and the $y$ axis is parallel to the surface. The plane of the page ( $y z$ plane) contains the reaction center, the center of the substituent dipole, and their corresponding images below the surface. This plane is perpendicular to the plane of the surface ( $x y$ plane). The molecular coordinates $\left(x, y^{\prime}, z^{\prime}\right)$ are rotated by an angle $\theta_{x}$ about the $x$ axis such that the $y^{\prime}$ axis lies along the charge-dipole vector, $\vec{r}$. There are five independent parameters used to describe the geometry of the point charge and the dipole over the surface. The parameter $z$ defines the position of the point charge over the surface, whereas the parameters $r$ and $\theta_{x}$ are required to define the dipole position with respect to the point charge. The angles $\phi$ and $\theta_{z}$ are the polar and azimuthal angles, respectively, 
TABLE 1: Definition of Variables Used in the Image Charge Model of a Point Charge and Dipole above a Conductive Surface Used To Analyze Substituent Effects on Metal Surfaces

\begin{tabular}{|c|c|c|}
\hline $\begin{array}{l}\text { independent } \\
\text { variable }\end{array}$ & \multicolumn{2}{|c|}{ description } \\
\hline$\Delta q$ & \multicolumn{2}{|c|}{$\begin{array}{c}\text { change in charge density of the reaction center } \\
\text { between reactant and transition state } \\
\text { (point charge) }\end{array}$} \\
\hline$\Delta \vec{\mu}$ & $\begin{array}{r}\text { difference of dipole vector be } \\
\text { different mo }\end{array}$ & $\begin{array}{l}\text { etween substituents of } \\
\text { lecules }\end{array}$ \\
\hline$z$ & $\begin{array}{r}\text { height of the point charge (reac } \\
\text { image pl }\end{array}$ & $\begin{array}{l}\text { ction center) above the } \\
\text { lane }\end{array}$ \\
\hline$\vec{r}$ & $\begin{array}{r}\text { vector between dipole vector } \\
\text { charg }\end{array}$ & $\begin{array}{l}\text { (substituent) and point } \\
\mathrm{e}\end{array}$ \\
\hline$\theta_{x}$ & $\begin{array}{r}\text { angle of the point charge-dipol } \\
\text { the } x \text { ax }\end{array}$ & $\begin{array}{l}\text { le vector with respect to } \\
\text { xis }\end{array}$ \\
\hline$\theta_{z}$ & $\begin{array}{r}\text { azimuthal angle of rotation of } \\
\text { the } z \text { ax }\end{array}$ & $\begin{array}{l}\text { the dipole vector about } \\
\text { xis }\end{array}$ \\
\hline$\phi$ & polar angle between dipole & vector and the $z$ axis \\
\hline $\begin{array}{l}\text { dependent } \\
\text { variable }\end{array}$ & description & equation \\
\hline$z_{\mu}$ & $\begin{array}{l}\text { height of the dipole above the } \\
\text { image plane }\end{array}$ & $z+r \sin \theta_{x}$ \\
\hline$r_{q_{\mathrm{r}} \mu_{\mathrm{i}}}$ & $\begin{array}{l}\text { distance between real charge and } \\
\text { image dipole }\end{array}$ & $\sqrt{4 z^{2}+r^{2}+4 r z \sin \theta}$ \\
\hline$r_{q_{\mathrm{i}}, \mu_{\mathrm{r}}}$ & $\begin{array}{l}\text { distance between image charge } \\
\text { and real dipole }\end{array}$ & $\sqrt{4 z^{2}+}$ \\
\hline
\end{tabular}

about the surface normal ( $z$ axis) that are used to define the orientation of the dipole in three-dimensional space $\left(\theta_{z}=0^{\circ}\right.$ is in the $y z$ plane). Three other dependent geometric variables are defined in order to simplify the potential energy equations: $z_{\mu}$,

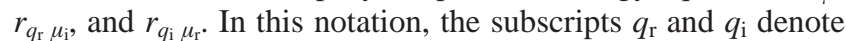
the real and image charges, repectively, whereas the subscripts $\mu_{\mathrm{r}}$ and $\mu_{\mathrm{i}}$ represent the real and image dipoles, respectively. A brief description of these dependent variables and their relationships to the independent variables is given in Table 1 . In the framework of these coordinate systems, a difference among substituent dipole vectors $(\Delta \vec{\mu})$ can be expressed as

$$
\begin{aligned}
\Delta \vec{\mu} & =\mu_{x} \hat{\imath}+\mu_{y} \hat{j}+\mu_{z} \hat{k} \\
& =\mu_{x} \hat{\imath}+\mu_{y^{\prime}} \hat{j}^{\prime}+\mu_{z^{\prime}} \hat{k}^{\prime}
\end{aligned}
$$

or

$$
\begin{array}{r}
\Delta \vec{\mu}=\mu_{x} \hat{\imath}+\left(\mu_{y^{\prime}} \cos \theta_{x}-\mu_{z^{\prime}} \sin \theta_{x}\right) \hat{j}+ \\
\left(\mu_{y^{\prime}} \sin \theta_{x}+\mu_{z^{\prime}} \cos \theta_{x}\right) \hat{k}
\end{array}
$$

The influence of the metal surface on substituent effects can be determined by considering the difference between the substituent effect in the gas phase $\left(\Delta \Delta E_{\mathrm{a}} \mathrm{g}\right)$ and the substituent effect on the surface $\left(\Delta \Delta E_{\mathrm{a}}{ }^{\mathrm{s}}\right)$. Using the geometry defined above, we will consider the potential energy resulting from the electrostatic interaction between the charge, the dipole, and their images. This can be compared to the gas-phase substituent effect, which simply arises from the interaction between the charge and the substituent dipole.

$$
\Delta \Delta E^{\mathrm{g}}=\left.\left(\Delta \Delta E_{q_{\mathrm{r}} \mu_{\mathrm{r}}}\right)\right|_{-\infty<z<\infty}=-\frac{1}{4 \pi \epsilon_{0}} \frac{\Delta q \Delta \vec{\mu} \cdot \hat{r}}{r^{2}}
$$

In this expression, $\Delta \vec{\mu} \cdot \hat{r}$ is the projection of the dipole onto the vector from the reaction center to the dipole $(\hat{r}$ is a unit vector along the direction of $\vec{r}$ ). The notation $\left.\right|_{-\infty<z<\infty}$ is simply a reminder that this energy of interaction is evaluated from the electric field over all space. The quantity $\epsilon_{0}$ is the permittivityof free space and has the value $8.85 \times 10^{-12} \mathrm{C}^{2}$ $\mathrm{N}^{-1} \mathrm{~m}^{-2}$. A similar charge-dipole term appears in the expression for the substituent effect on the surface, although it is evaluated considering only the electric field in the half-space above the conducting surface.

The effect of the metal surface on an electrostatic substituent effect is due to the interactions of the real charge and dipole with the image charge and dipole. There are several electrostatic interactions that determine the energy of the charge-dipole complex over a conducting surface. To determine the substituent effect on an activation energy, one measures the difference in activation energy between two molecules having different substituent dipoles.

$$
\Delta \Delta E^{\mathrm{s}}=\left(E_{\mathrm{R}}^{\ddagger}-E_{\mathrm{R}}^{\mathrm{r}}\right)-\left(E_{0}^{\ddagger}-E_{0}^{\mathrm{r}}\right)
$$

The subscripts refer to the substituted $\left(E_{\mathrm{R}}\right)$ and unsubstituted $\left(E_{0}\right)$ molecules, and the superscripts refer to the reactant $\left(E^{\mathrm{r}}\right)$ and the transition state $\left(E^{\ddagger}\right)$. Each of these energies has several contributions from the electrostatic interactions of the charge and dipole with themselves and with their images. The charge change at the reaction center, $\Delta q$, on going from reactant to transition state, is considered to be the same for all substituted molecules. Because the interaction between the reaction center and its image charge does not change when molecules with different substituents are compared, this interaction does not influence the substituent effect and can be ignored. Also, the interaction between the dipole and its image is assumed to be the same for the reactant and transition state. As a result, it does not influence the activation energy. Thus, in considering the influence of the surface on substituent effects, one is only concerned with electrostatic interactions involving both a charge (real or image) and a dipole (real or image).

$$
\begin{array}{r}
\Delta \Delta E^{\mathrm{s}}=\left.\left(\Delta \Delta E_{q_{\mathrm{r}} \mu_{\mathrm{r}}}\right)\right|_{z>0}+\left.\left(\Delta \Delta E_{q_{\mathrm{i}} \mu_{\mathrm{i}}}\right)\right|_{z>0}+\left.\left(\Delta \Delta E_{q_{\mathrm{r}} \mu_{\mathrm{i}}}\right)\right|_{z>0}+ \\
\left.\left(\Delta \Delta E_{q_{\mathrm{i}} \mu_{\mathrm{r}}}\right)\right|_{z>0}
\end{array}
$$

The $\left.\right|_{z>0}$ notation is a reminder that the interaction energy is calculated using only the electric field above the surface. When using the method of images to calculate energy, one must remember that there is no electric field below the metal surface. As a result, although it takes work to move the real charge from $z=\infty$ into position at a height " $z$ " above the surface, no work is needed to move the image charge below the surface. For example, the energy of a point charge interacting with its image is one-half the energy of two real charges at the same separation.

In considering the individual terms in the expression for the substituent effect on the surface, it is fairly easy to see that the (real charge)-(real dipole) and (image charge)-(image dipole) terms are equivalent to the charge-dipole interaction in the gasphase reaction. From symmetry, it can be seen that

$$
\left.\left(\Delta \Delta E_{q_{\mathrm{r}} \mu_{\mathrm{r}}}\right)\right|_{z>0}+\left.\left(\Delta \Delta E_{q_{\mathrm{i}} \mu_{\mathrm{i}}}\right)\right|_{z>0}=\left.\left(\Delta \Delta E_{q_{\mathrm{r}} \mu_{\mathrm{r}}}\right)\right|_{-\infty<z<\infty}
$$

This equality exists because, when the charge and dipole are positioned near the surface, the electric field that would have existed below the surface $(z<0)$ if it were not conductive is exactly the field that exists above the surface as a result of the images. The influence of the metal surface on the substituent effect is then given by

$$
\Delta \Delta E^{\mathrm{s}}-\Delta \Delta E^{\mathrm{g}}=\left.\left(\Delta \Delta E_{q_{\mathrm{r}}} \mu_{\mathrm{i}}\right)\right|_{z>0}+\left.\left(\Delta \Delta E_{q_{\mathrm{i}} \mu_{\mathrm{r}}}\right)\right|_{z>0}
$$

If one hypothetically ignores the surface, these interactions are symmetric. The distances $r_{q_{\mathrm{r}}} \mu_{\mathrm{i}}$ and $r_{q_{\mathrm{i}} \mu_{\mathrm{r}}}$ are equal, and the signs 
of the interactions are the same. Because these are image interactions, the energy is one-half what it would be if they were all real. By symmetry,

$$
\begin{aligned}
\Delta \Delta E_{q_{\mathrm{r}} \mu_{\mathrm{i}}}+\Delta \Delta E_{q_{\mathrm{i}} \mu_{\mathrm{r}}} & =-\left(\frac{1}{2}\right) \frac{1}{4 \pi \epsilon_{0}}\left(\frac{\Delta q \Delta \vec{\mu} \cdot \hat{r}_{q_{\mathrm{r}} \mu_{\mathrm{i}}}}{r_{q_{\mathrm{r}} \mu_{\mathrm{i}}}^{2}}+\frac{\Delta q \Delta \vec{\mu} \cdot \hat{r}_{q_{\mathrm{i}} \mu_{\mathrm{r}}}}{r_{q_{\mathrm{i}}, \mu_{\mathrm{r}}}^{2}}\right) \\
& =-\frac{1}{4 \pi \epsilon_{0}} \frac{\Delta q \Delta \vec{\mu} \cdot \hat{r}_{q_{\mathrm{r}} \mu_{\mathrm{i}}}}{r_{q_{\mathrm{r}} \mu_{\mathrm{i}}}^{2}}
\end{aligned}
$$

where

$$
\hat{r}_{q_{\mathrm{i}} \mu_{\mathrm{r}}}=\frac{\left(r \cos \theta_{x}\right) \hat{j}+\left(2 z+r \sin \theta_{x}\right) \hat{\mathrm{k}}}{r_{q_{\mathrm{i}} \mu_{\mathrm{r}}}}
$$

An item to note is that, because the $\mu_{x}$ component of the substituent dipole is perpendicular to $\vec{r}$ and to $\hat{r}_{q_{i} \mu_{r}}$, there is no interaction between $\mu_{x}$ and either the charge or its image. Thus, if the dipole is out of the plane $\left(\theta_{z} \neq 0\right)$, it would only be necessary to include its projection onto the $y z$ plane in the model. This single expression, then, gives the magnitude of the influence of the surface on the substituent effect, within the assumptions and limitations of this image charge model.

It should be noted that the model that we describe is for an isolated reactant on a metal surface and that it ignores interactions between the reacting molecule and any background of other adsorbed molecules that would be present on the surface at high coverages. The coverage dependence of the interactions between adsorbed dipoles and their images has been discussed in detail by Maschhoff and Cowin. ${ }^{18}$ We are justified in using the lowcoverage model by the fact that we have not observed experimentally any coverage dependence in the barriers to $\beta$-hydride elimination in the ethoxides on the $\mathrm{Cu}(111)$ surface.

\section{Results}

The model described above will be used to analyze the substituent effect for an important surface reaction, $\beta$-hydride elimination from ethoxide on $\mathrm{Cu}(111)$. Ethoxides with varying degrees of fluorine substitution at the methyl group have been used to determine the nature of the transition state for $\beta$-hydride elimination. Measurements of an increase in activation energy with increasing fluorine substitution have shown that the transition state is electron deficient with respect to the ethoxide. ${ }^{4}$ For the current analysis, only two molecules will be compared: trifluoroethoxide $\left(\mathrm{CF}_{3} \mathrm{CH}_{2} \mathrm{O}_{\mathrm{ad}}\right)$ and ethoxide $\left(\mathrm{CH}_{3} \mathrm{CH}_{2} \mathrm{O}_{\mathrm{ad}}\right)$. These two reactants exhibit a substantial substituent effect $\left(\Delta \Delta E_{\mathrm{a}}^{\mathrm{s}}=55 \mathrm{~kJ} / \mathrm{mol}\right)$, and the orientations of these two molecules on the $\mathrm{Cu}(111)$ surface have also been measured..$^{9,10}$ The magnitude of the difference in the dipole moments of the methyl and trifluoromethyl groups is approximated by the magnitude of the dipole of trifluoromethane $\left(\mathrm{CF}_{3} \mathrm{H}\right)$ as $\Delta \vec{\mu}_{y^{\prime}}=$ $-5.5 \times 10^{-30} \hat{j}^{\prime} \mathrm{C} \cdot \mathrm{m}$, and the resulting dipole moment vector is assumed to be aligned along the $\mathrm{C}-\mathrm{C}$ bond of the ethoxide, $\theta_{z}=0{ }^{\circ} .{ }^{17}$ For this choice of geometry, the energy change that results from the influence of the surface can be expressed as

$$
\Delta \Delta E^{\mathrm{s}}-\Delta \Delta E^{\mathrm{g}}=\frac{1}{4 \pi \epsilon_{0}} \frac{\Delta q \Delta \mu_{y^{\prime}}}{r_{q_{\mathrm{i}} \mu_{\mathrm{r}}}^{2}}\left(\frac{r+2 z \sin \theta_{x}}{r_{q_{\mathrm{i}} \mu_{\mathrm{r}}}}\right)
$$

The value of $\theta_{x}=30^{\circ} \pm 10^{\circ}$ is obtained from the literature and is chosen as the average orientation between $\mathrm{CH}_{3} \mathrm{CH}_{2} \mathrm{O}_{\mathrm{ad}}$ and $\mathrm{CF}_{3} \mathrm{CH}_{2} \mathrm{O}_{\text {ad. }}{ }^{9,10}$ Because the dipole moment lies along the
$\mathrm{C}-\mathrm{C}$ bond such that the positive end is toward the reaction center, there is a relationship between $\theta_{x}$ and $\phi$ such that $\phi=$ $90^{\circ}+\theta_{x}$, or $\phi=120^{\circ} \pm 10^{\circ}$. The change in charge at the reaction center, $\Delta q$, is chosen to have a magnitude equal to three-eighths of an elementary charge or $0.6 \times 10^{-19} \mathrm{C}$ and to be located at the $\beta$-C atom. The choice of $\Delta q=3 /{ }_{8} e$ gives a value of $\Delta \Delta E^{\mathrm{g}}=55 \mathrm{~kJ} / \mathrm{mol}$ for the substituent effect, which is of the same magnitude as that measured experimentally. A charge change of $1 /{ }_{2} e$ would represent the extreme case of a bond going from completely covalent to completely ionic. The distance between the point charge and the dipole, $r$, is approximated by the length of a $\mathrm{C}-\mathrm{C}$ bond plus one-half the length of the projection of a $\mathrm{C}-\mathrm{F}$ bond of the trifluoromethyl group onto the $\mathrm{C}-\mathrm{C}$ bond axis, specifically, $r=1.8 \AA{ }^{17}$ The appropriate choice for the height of the reaction center above the surface, $z$, is the parameter that is the most difficult to determine precisely. One part of the difficulty is the fact that no direct measurements have been made of the $\mathrm{Cu}-\mathrm{O}$ bond length in ethoxides or the location of the oxygen atom with respect to the surface atoms. The geometry of methoxy on $\mathrm{Cu}$ (111), on the other hand, has been measured. ${ }^{14}$ Methoxy is found to be located in the threefold hollow site with the $\beta$-C atom positioned $2.8 \AA$ above the surface atom plane. ${ }^{19}$ It is likely, however, that ethoxide is even further from the surface as a result of the steric effects of the larger methyl group. Another unknown in the model is the position of the image plane with respect to the real metal surface. On the basis of the uniform background model mentioned earlier, the image plane is approximated as lying $1.8 \AA$ above the plane defined by the nuclei of the surface atoms. Therefore, we estimate the height of the reaction center of ethoxide and trifluoroethoxide at 1-2 $\AA$ above the image plane. In the results discussed below, the height of the reaction center above the image plane is allowed to vary in order to illustrate the sensitivity of the substituent effect to its position with respect to the image plane. By calculating the effects of the surface on the substituent effects for various geometries of the dipole and reaction center, we can obtain some feeling for the influence of the surface on the substituent effect.

The image charge theory model described above will be used to evaluate the influence of the metal surface on the substituent effect measurements for $\beta$-hydride elimination on $\mathrm{Cu}(111)$. We have calculated the effect of the metal on the energy of a charge and a dipole above a conductive surface at several geometries. A plot of the substituent effect on the metal surface, $\Delta \Delta E^{\mathrm{s}}$, versus the height of the reaction center above the image plane, $z$, at several different values of $\theta_{x}\left(0^{\circ}, 15^{\circ}, 30^{\circ}, 60^{\circ}\right.$, and $\left.90^{\circ}\right)$ is shown in Figure 2. In this plot, the values of $\Delta \vec{\mu}_{y^{\prime}}=-5.5$ $\times 10^{-30} \hat{j} \mathrm{C} \cdot \mathrm{m}, \Delta q=0.6 \times 10^{-19} \mathrm{C}, r=1.8 \AA, \theta_{z}=0^{\circ}$, and $\phi=90^{\circ}+\theta_{x}$ (aligned with $\mathrm{C}-\mathrm{C}$ bond) are chosen to approximate $\beta$-hydride elimination in ethoxide on the $\mathrm{Cu}(111)$ surface. The dashed curve of $\theta_{x} \approx 30^{\circ}$ corresponds to the known orientation of ethoxide on $\mathrm{Cu}(111)$. It can be seen that the substituent effect is only slightly different from the gas-phase value, $<25 \%$, at all heights greater than $1 \AA$. The effect of the metal is to reduce the substituent effect slightly from the gasphase value. It is clear that, although the metal can alter the magnitude of the substituent effect, it is highly unlikely that the metal will completely screen substituent effects, given that the reaction center and the substituent are above the image plane.

On a metal surface, the orientation of the adsorbate can also have an impact on the substituent effect. The value of $\Delta \Delta E^{\mathrm{s}}$ as a function of $\theta_{x}$ at several fixed heights is shown in Figure 3. All other parameters were the same as those used to produce 


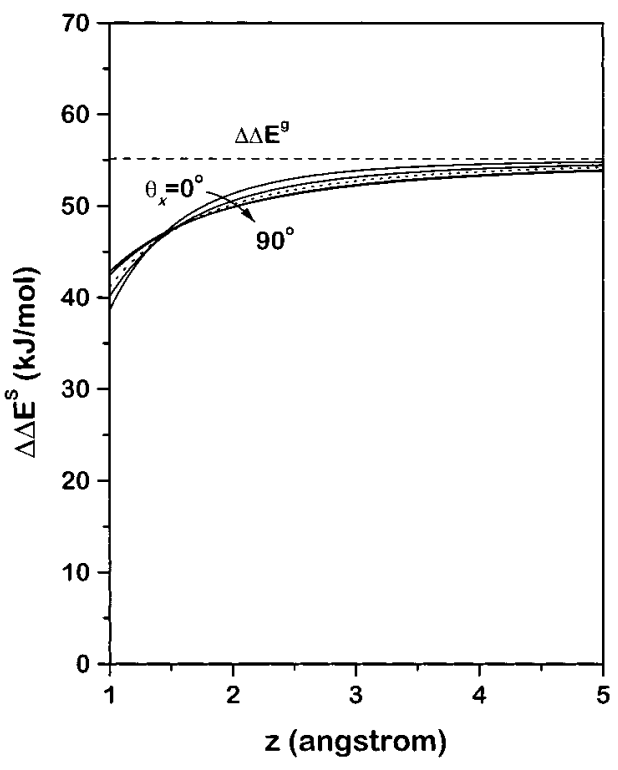

Figure 2. Plot of the substituent effect $\left(\Delta \Delta E^{\mathrm{s}}\right)$ versus the height of a dipole substituent $(\Delta \mu)$ and a point charge reaction center $(\Delta q)$ at various constant values of $\theta_{x}\left(0^{\circ}, 15^{\circ}, 30^{\circ}, 60^{\circ}\right.$, and $\left.90^{\circ}\right)$. The value of $\Delta \Delta E^{\mathrm{s}}$ is within $25 \%$ of $\Delta \Delta E^{\mathrm{g}}(55 \mathrm{~kJ} / \mathrm{mol})$ at all heights above $1 \AA . \Delta q$ $=0.6 \times 10^{-19} \mathrm{C}, \Delta \mu=5.5 \times 10^{-30} \mathrm{C} \cdot \mathrm{m}, r=1.8 \AA, \theta_{z}=0^{\circ}$, and $\phi$ $=90^{\circ}+\theta_{x}$.
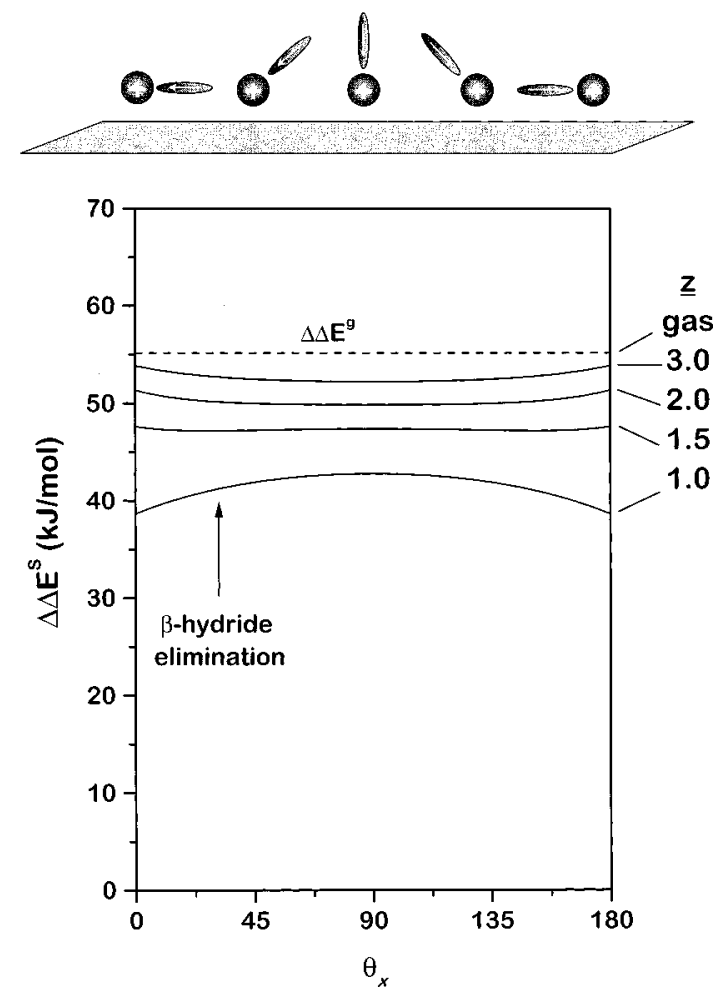

Figure 3. Plot of the substituent effect versus the angle of rotation, $\theta_{x}$, of the dipole about the reaction center at different heights, $z$, above a conductive surface. The values of $r=1.8 \AA$ and $\theta_{z}=0^{\circ}$ were chosen to represent the orientation of ethoxide on $\mathrm{Cu}(111)$ and were held constant. The value of $\theta_{x}=30^{\circ}$ for ethoxide is shown with an arrow. At all geometries where the dipole is further from the surface than the reaction center $\left(0^{\circ}<\theta_{x}<180^{\circ}\right)$, the surface substituent effect is only slightly less than the gas-phase substituent effect.

Figure 2 and represent the orientation of ethoxide or trifluoroethoxide on $\mathrm{Cu}(111)$. It can be seen in Figure 3 that, at any given height, the substituent effect is not greatly affected by the orientation and that, at heights above $1 \AA$, the value $\Delta \Delta E^{\mathrm{s}}$ differs by $<25 \%$ from $\Delta \Delta E^{\mathrm{g}}$. In the case of ethoxide, the value of $z$ is deemed to lie in the range $1.5-2 \AA$.

\section{Discussion}

The goal of this work is to determine the effect that metal surfaces have on substituent effects for reactions on metal surfaces. The substituent effect for $\beta$-hydride elimination in ethoxides has been measured on the $\mathrm{Cu}(111)$ surface and has been demonstrated to have a magnitude that parallels the energetics of gas-phase alcohol dehydrogenation. From these image charge theory calculations, we discover that the metal surface should, in fact, have little effect on the energetics of $\beta$-hydride elimination. We also find that the metal surface will, in general, only be expected to have a significant effect for surface reactions in which the substituent and reaction center are very close $(<1 \AA)$ to the image plane. Thus, for many metal surface reactions, the metal does not significantly screen the substituent effect.

The model presented for the analysis of the influence of the metal surface on substituent effects makes several unavoidable assumptions that should be clearly described. The suggestion that the charge change at the reaction center $(\Delta q)$ is the same for both substituted and unsubstituted molecules is no different from that made in the analysis of the gas-phase substituent effects. This is also true of the suggestion that the substituent dipole moment does not change on going from reactant to transition state. The major assumption that is made here is that the interactions of real charges and dipoles with their images are the same in reactants and transition states and in substituted and unsubstituted adsorbates. Without knowledge of the details of the structures of these species, this assumption cannot be avoided. Probably the most serious unknown in this problem is the position of the image plane. It certainly lies well outside the plane defined by the atomic nuclei. ${ }^{13-16}$ Electronic structure calculations show that the position of the image plane can be considered to vary with the position of a charge over a surface. In reality, the image charge model becomes questionable at the distances from the surface at which reactions occur. For the purposes of this work, the concept of the image plane serves as a first-order model for analyzing the effects of the surface.

Because the metal does not negate substituent effects, measurements of substituent effects can be a valuable tool for obtaining insight into the electronic nature of reactions on metal surfaces. Perhaps more importantly, these calculations indicate that energy measurements made in the gas phase may provide valuable information for the energetics of metal surface reactions. Another question that arises in using substituent effects to determine the nature of transition states for surface reactions is which substituent constants should be used to determine linear free-energy relationships. There are currently no tabulated substituent constants for surface reactions, only for solutionphase and gas-phase constants. Because the metal surface is not likely to alter the energetics significantly from the gas phase, the gas-phase substituent constants provide a logical choice.

Acknowledgment. This work was supported by NSF Grant CHE-9701924. M.T.B. was supported by a fellowship from the Shell Foundation.

\section{References and Notes}

(1) Taft, R. W.; Topsom, R. D. Prog. Phys. Org. Chem. 1987, 16, 1.

(2) Hansch, C.; Leo, A.; Taft, R. W. Chem. Rev. 1991, 91, 165.

(3) Wells, P. R. Linear Free Energy Relationships; Academic Press: New York, 1968 
(4) Gellman, A. J.; Dai, Q. J. Am. Chem. Soc. 1993, 115 (2), 714.

(5) Forbes, J. G.; Gellman, A. J. J. Am. Chem. Soc. 1993, 115 (14), 6277.

(6) Meyers, J. M.; Gellman, A. J. Surf. Sci. 1995, 337, 40.

(7) Zhou, G.; Chan, C.; Gellman, A. J. J. Phys. Chem. B 1999, 103 (7), 1134.

(8) Buelow, M. T.; Zhou, G.; Immaraporn, B.; Gellman, A. J. Catal. Lett. 1999, 59, 9 .

(9) Street, S. C.; Gellman, A. J. J. Chem. Phys. 1996, 105 (16), 7158

(10) Street, S. C.; Gellman, A. J. J. Phys. Chem. 1996, 100 (20), 8338

(11) Gellman, A. J.; Buelow, M. T.; Street, S. C.; Morton T. H. J. Phys. Chem. In press.
(12) Griffiths, D. J. Introduction to Electrodynamics, 2nd ed.; Prentice Hall: Engelwood Cliffs, NJ, 1989.

(13) García-Hernández, M.; Bagus, P. S.; Illas, F. Surf. Sci. 1998, 409, 69.

(14) Lang, N. D.; Kohn, W. Phys. Rev. B 1970, 1, 4555.

(15) Lang, N. D.; Kohn, W. Phys. Rev. B 1971, 3, 1215.

(16) Lang, N. D.; Kohn, W. Phys. Rev. B 1973, 7, 3541.

(17) CRC Handbook of Chemistry and Physics, 78th ed.; Lide, D. R., Ed.; CRC Press: Boca Raton, FL, 1997.

(18) Maschhoff, B. L.; Cowin, J. P. J. Chem. Phys. 1994, 101 (9), 8138

(19) Hofmann, Ph.; Schindler, K.-M.; Bao, S.; Firtzche, V.; Ricken, D. E.; Bradshaw, A. M.; Woodruff, D. P. Surf. Sci. 1994, 304 (1-2), 74. 